\title{
Frequency of Carbapenemase Producing Klebsiella pneumoniae in Makkah, Saudi Arabia
}

\author{
Mohammad Mubashir Ahmad Khan ${ }^{1}$, Aftab Faiz ${ }^{2}$ \\ ${ }^{1}$ Department of Laboratory Medicine, Faculty of Applied Medical Sciences, Umm Al Qura University, Makkah, Saudi Arabia \\ ${ }^{2}$ Maternity and Children Hospital, Makkah, Saudi Arabia
}

\begin{abstract}
Objective: Carbapenemase producing Klebsiella pneumoniae is emerging as an important pathogen worldwide which has alarming clinical threat with serious effects on patients' outcome. The aim of our study is to determine prevalence of carbapenemase producing K. pneumoniae and its antibiotic susceptibility pattern in tertiary care hospitals.

Methods: A prospective study was performed on 260 clinical isolates of $K$. pneumoniae collected from five different hospitals of Makkah, Saudi Arabia from January to July 2015. All clinical isolates were confirmed as K. pneumoniae using Vitek 2 system. These isolates were then screened for potential carbapenemase producers by determining reduced susceptibility to carbapenems using representative antibiotic disks of third generation cephalosporins and carbapenems in the disk diffusion test following guidelines of Clinical and Laboratory Standards Institute. Minimum inhibitory concentration of isolates found positive in screening test were determined for cephalosporins, carbapenems, aminoglycosides and tigecycline and colistin using Vitek-2 system. Modified Hodge test was performed to detect carbapenemase production on the isolates suspected for carbapenemase production.
\end{abstract}

Results: Out of $260 \mathrm{~K}$. pneumoniae isolates, 31 (11.9\%) have shown 100\% resistance to all cephalosporins. Of $31 \mathrm{~K}$. pneumoniae isolates, 15 (48.4\%) were found positive for carbapenemase. All carbapenem resistant isolates were sensitive to colistin and tigecycline. However, these isolates have shown resistance to amikacin (41.9\%) and gentamicin (51.6\%), respectively.

Conclusion: This is the first report from Makkah reporting carbapenamse producing K.pneumoniae. Of $12 \%$ potential carbapenemase producing K.pneumoniae, $48.4 \%$ were found positive for carbapenemase production. Molecular characterization of these strains will help to determine the type of carbapenemase prevalent in this area. J Microbiol Infect Dis 2016;6(3): 121-127

Key words: Klebsiella pneumoniae; carbapenemase; drug resistance; Modified Hodge test

\section{INTRODUCTION}

Carbapenems are usually used to treat infections caused by Gram-negative bacteria that produce extended-spectrum $\beta$-lactamases. Multiple drug resistant and carbapenemase producing $K$. pneumoniae is emerging as an important pathogen worldwide, which has alarming clinical threat with serious effects on patient outcomes [1]. According to global antibiotic resistance estimates published by the World Health Organization in 2014, Klebsiella pneumoniae and Escherichia coli were listed as two of the three agents of greatest concern, associated with both hospital and community acquired infections [2].
Among the Enterobacteriaceae, clinically significant carbapenemases are the Ambler molecular class A (KPC), class B (VIM, IMP, NDM), and class D (OXA-48) types [3]. Among them, two clinically significant carbapenemase subclasses; $K$. pneumoniae carbapenemase (KPC) and New Delhi metallo- $\beta$-lactamase-1 (NDM-1) are of particular importance [4].

The KPC which was first identified in 2001 in USA [5] remained limited to USA till 2005. Since then, the spread of KPC has accelerated worldwide and KPC-producing strains are now emerging worldwide $[6,7]$ and also become endemic in many parts of the world, including the United States,

Correspondence: Mohammad Mubashir Ahmad Khan, PhD, Professor, Department of Laboratory Medicine, Faculty of Applied Medical Sciences, Umm Al Qura University, P.O. Box. 7607, Makkah, Saudi Arabia Email: mubashirpmrc@yahoo.com Received: 26.01.2016, Accepted: 10.05.2016

Copyright (C) Journal of Microbiology and Infectious Diseases 2016, All rights reserved 
Greece, South America, and China [8]. The NDM1 was first identified in 2008 in a K. pneumoniae isolate recovered from a Swedish patient who has been previously hospitalized in New Delhi, India [9]. Soon after this, NDM-1 was found throughout India, Pakistan, and in the United Kingdom [10] and are increasingly being reported worldwide [11]. Although, India was considered as the main reservoir of the NDM-1- producers, several NDM-1-producing enterobacterial isolates have been reported from the Balkan states [12] suggesting that this is another reservoir of NDM-1 producers.

Recent studies conducted in Middle East countries also suggests that this region might be an additional reservoir of NDM producers as it has been reported from Oman [13], Lebanon [14], Saudi Arabia [15,16], and Qatar [17].

The Modified Hodge Test (MHT) has been used extensively as a phenotypic method for the detection of carbapenemase activity and it is the only carbapenemase detection method recommended by the Clinical and Laboratory Standard Institute (CLSI) for screening purposes [18]. Molecular detection of carbapenemase genes using polymerase chain reaction (PCR) based methods was found to be quick, accurate and effective in the detection of specific carbapenemases genes, such as KPC, NDM-1, OXA-48, etc. [13-17]. Simple PCR assays targeting a single carbapenemase type have been used successfully in numerous studies. It has also been reported that PCR tests have the highest specificity in identification and confirmation of the carbapenemases [5,10,12].

In coming years, carbapenemase producing $K$. pneumoniae can cause considerable clinical problems because they are multidrug resistant, lacking susceptibility to $\beta$-lactam antibiotics, fluoroquinolones, and aminoglycosides. Thus, therapy for clinically significant isolates rests on the use of tigecycline or polymyxins (colistin), both of which have been associated with the development of resistance and are potentially toxic [19].

Since carbapenemase producing K. pneumoniae is an emerging pathogen worldwide which has serious clinical and infection control implications, therefore, this study was designed with the objective to determine the frequency of carbapenemase producing $K$. pneumoniae in various tertiary care hospitals.

\section{METHODS}

\section{Bacterial isolates}

This prospective descriptive study was carried out from January to July 2015 at Microbiology Laboratory of Department of Laboratory Medicine, Faculty of Applied Medical Sciences, Umm Al Qura University, Makkah, Saudi Arabia after the approval of institutional Bioethical Committee. A total of 260 $K$. pneumoniae clinical isolates were collected from five different hospitals of Makkah city during the study period. The distribution of these isolates among participating hospitals was: Al-Noor Specialist hospital ( $n=92)$; King Faisal hospital $(n=50)$; King Abdul Aziz hospital ( $n=45$ ); Hera'a General hospital $(n=36)$; Maternity and Children hospital $(n=37)$.

\section{Identification of clinical isolates as $K$. pneumoniae}

All clinical isolates collected from the hospitals were identified using standard microbiological methods. Briefly, isolates were sub cultured on blood agar plates and incubated at $37^{\circ} \mathrm{C}$ for $16-24$ hours. Following incubation, colonies were processed for identification of $K$. pneumoniae using Vitek $2 \mathrm{com}$ pact system (BioMerieux, USA).

\section{Screening of $K$. pneumoniae isolates for suspected carbapenemase production}

All K. pneumoniae isolates were first screened for reduced susceptibility to carbapenems by disk diffusion method following guidelines of CLSI [18]. Following antibiotic disks (Oxoid, UK) were used in the disk diffusion susceptibility test: ceftazidime $(30 \mu \mathrm{g})$, ceftriaxone $(30 \mu \mathrm{g})$, cefepime $(30 \mu \mathrm{g})$, imipenem (10 $\mu \mathrm{g})$ and meropenem $(10 \mu \mathrm{g})$. Using standard interpretive table of zone diameter breakpoint of CLSI, the test organism was classified as susceptible, intermediate or resistant.

According to CLSI guidelines K. pneumoniae that are resistant to one or more of the third generation cephalosporins e.g., cefotaxime, ceftriaxone, and/or ceftazidime and have reduced zones of sensitivity to carbepenem (e.g. ertapenem 19-21 $\mathrm{mm}$ and/or meropenem 16-21 $\mathrm{mm}$ ) are suspected for carbapenemase production. Alternately, MICs 2 $\mu \mathrm{g} / \mathrm{ml}$ for ertapenem and 2-4 $\mu \mathrm{g} / \mathrm{ml}$ for meropenem may indicate carbapenemase production. K. pneumoniae isolates which fulfilled these criteria were subjected to MHT for the detection of carbapenemase production. 
Detection of carbapenemase production in suspected isolates

All suspected isolates (from screening step) of $K$. pneumoniae were tested for the production of carbapenemase by MHT as described in CLSI guidelines [18] and results were interpreted accordingly. The MHT was performed as follows: First of all bacterial suspension of the carbapenem susceptible strain of E.coli ATCC 25922 was prepared in $5 \mathrm{ml}$ sterile saline and turbidity was adjusted to 0.5 McFarland using turbidity meter Densicheck plus (BioMerieux). This suspension was then diluted to 1:10 using sterile saline. This diluted suspension of E.coli ATCC 25922 was then swabbed onto labeled Mueller Hinton agar plate. The plate was allowed to dry for 3-5 minutes. Then, a $10 \mu \mathrm{g}$ meropenem susceptibility disk was placed in the centre of the plate. K. pneumoniae ATCC BAA 1705 and K. pneumoniae ATCC BAA 1706 were used as positive and negative control, respectively. After this, positive and negative controls and test microorganisms were streaked in a straight line from the edge of the disk to the edge of the plate. The plate was then incubated at $37^{\circ} \mathrm{C}$ for $16-18$ hours. After this incubation period, plate was examined for a cloverleaf-like indentation at the joining of the test organism and the E. coli ATCC 25922, within the zone of inhibition of the carbapenem susceptibility disk.

A positive MHT test had a clover leaf-like indentation of the E. coli ATCC 25922 growing along the test organism growth streak within the zone of inhibition because the carbapenemase produced by $K$. pneumoniae isolate inactivated meropenem that diffused into the medium. Thus there was not sufficient meropenem present to inhibit the growth of E. coli
ATCC 25922 and indentation of zone was noted. A positive MHT indicated carbapenemase production by the test microorganism. A negative MHT test has no growth of the E. coli ATCC 25922 along the test organism growth streak within the zone of inhibition. A negative test indicated no carbapenemase production by the test microorganism.

\section{Antibiotic susceptibility test of carbapenem resistant isolates}

Minimum inhibitory concentration of isolates which were found positive in screening test were determined for ceftazidime, ceftriaxone, cefepime, imipenem, meropenem, amikacin, gentamicin, tigecycline and colistin using gram-negative antibiotic susceptibility panel AST-N292 of Vitek-2 system (BioMerieux, USA). The data of all isolates was entered and analyzed using Microsoft Excel 2007.

\section{RESULTS}

\section{K. pneumoniae isolates screened for suspected carbapenemase production}

In this study a total of $260 \mathrm{~K}$. pneumoniae strains isolated at five different hospitals of Makkah were screened for potential carbapenemase producers using third generation cephalosporins and carbapenem disks. The drug resistance to scephalosporin ranged from $29.2 \%$ to $42.7 \%$, whereas among carbapenems; both imipenem and meropenem showed $11.9 \%$ resistance, each (Table 1). Among cephalosporins, ceftriaxone showed highest resistance $(42.7 \%)$ followed by cefepime $(30.9 \%)$ and ceftazidime $(29.2 \%)$. Out of 260 K. pneumoniae isolates $31(11.9 \%)$ isolates showed $100 \%$ resistance to all cephalosporins and carbapenems tested.

Table 1. Screening results of K. pneumoniae isolates for potential carbapenemase production.

\begin{tabular}{lcccccc}
\hline Hospitals & CFZ & CTX & CFP & IMP & MRN & Pan resistance \\
\hline Al-Noor Specialist Hospital $(n=92)$ & $29(31.5)$ & $42(45.6)$ & $25(27.2)$ & $13(14.1)$ & $13(14.1)$ & $13(14.1)$ \\
\hline King Faisal Hospital $(n=50)$ & $15(30.0)$ & $29(58.0)$ & $11(22.0)$ & $3(6.0)$ & $3(6.0)$ & $3(6.0)$ \\
King Abdul Aziz Hospital $(n=45)$ & $16(35.5)$ & $21(46.6)$ & $14(31.1)$ & $10(22.2)$ & $10(22.2)$ & $10(22.2)$ \\
\hline Hera'a General Hospital $(n=36)$ & $12(33.3)$ & $16(44.4)$ & $12(33.3)$ & $3(8.3)$ & $3(8.3)$ & $3(8.3)$ \\
\hline Maternity and Children Hospital $(n=37)$ & $4(10.8)$ & $3(8.1)$ & $3(8.1)$ & $2(5.4)$ & $2(5.4)$ & $2(5.4)$ \\
\hline Overall drug resistance $(n=260)$ & $76(29.2)$ & $111(42.7)$ & $65(30.9)$ & $31(11.9)$ & $31(11.9)$ & $31(11.9)$ \\
\hline
\end{tabular}

CFZ=Ceftazidime, CTX=Ceftriaxon, CFP=Cefepim, IMP=Imipenem, MER=Meropenem, Pan resistance=Pan-resis tance to pan resistance and add cephalosporins and carbapenems 
Table 2. Minimum inhibitory concentration of carbapenem resistant K. pneumoniae and results of Modified Hodge Test.

\begin{tabular}{|c|c|c|c|c|c|c|c|c|c|c|}
\hline $\begin{array}{l}\text { Isolate } \\
\text { No }\end{array}$ & CFZ & CTX & CEF & IMP & MER & AK & GEN & COL & TIG & MHT \\
\hline C-1 & $>64$ & $>64$ & $>64$ & $>16$ & $>16$ & 16 & 4 & $<0.5$ & $<0.5$ & $+v e$ \\
\hline C-2 & $>16$ & $>64$ & $>16$ & $>8$ & $>8$ & $<16$ & $>8$ & $<0.5$ & $<0.5$ & $+v e$ \\
\hline C-3 & $>64$ & $>64$ & $>64$ & $>16$ & $>16$ & 16 & $<1$ & $<0.5$ & $<0.5$ & -ve \\
\hline C-4 & $>64$ & $>64$ & $>64$ & $>16$ & $>16$ & 16 & $<1$ & $<2$ & 2 & $+\mathrm{ve}$ \\
\hline$C-5$ & $>64$ & $>64$ & $>64$ & $>16$ & $>16$ & 16 & $<1$ & $<0.5$ & $<0.5$ & $+v e$ \\
\hline C-6 & $>64$ & $>64$ & $>64$ & $>16$ & $>16$ & 16 & $<1$ & $<0.5$ & $<0.5$ & $+\mathrm{ve}$ \\
\hline C-7 & $>64$ & $>64$ & $>64$ & $>16$ & $>16$ & 16 & $<1$ & $<0.5$ & $<0.5$ & $+v e$ \\
\hline C-8 & $>16$ & $>64$ & $>64$ & $>16$ & $>16$ & 16 & 4 & $<0.5$ & $<0.5$ & $+v e$ \\
\hline C-9 & $>16$ & $>64$ & $>64$ & $>16$ & $>16$ & 16 & 4 & $<0.5$ & 2 & -ve \\
\hline C-10 & $>64$ & $>64$ & $>64$ & 8 & $>16$ & $>64$ & $>16$ & $<2$ & 2 & -ve \\
\hline C-11 & $>64$ & $>64$ & $>64$ & 8 & $>16$ & $>64$ & $>16$ & $<2$ & 2 & -ve \\
\hline C-12 & $>64$ & $>64$ & $>64$ & $>16$ & $>16$ & $>64$ & $>16$ & $<0.5$ & $<0.5$ & -ve \\
\hline C-13 & $>64$ & $>64$ & $>64$ & $>16$ & $>16$ & $>64$ & $>16$ & $<0.5$ & $<0.5$ & -ve \\
\hline C-14 & $>64$ & $>64$ & $>64$ & 4 & $>16$ & $>64$ & $>16$ & $<0.5$ & $<0.5$ & -ve \\
\hline C-15 & $>64$ & $>64$ & $>64$ & $>16$ & $>16$ & $>64$ & $>16$ & 2 & 2 & -ve \\
\hline C-16 & $>64$ & $>64$ & $>64$ & 8 & $>16$ & $>64$ & $>16$ & $<0.5$ & $<0.5$ & -ve \\
\hline C-17 & $>64$ & $>64$ & $>64$ & $>16$ & $>16$ & $>64$ & $>16$ & $<0.5$ & $<0.5$ & -ve \\
\hline C-18 & $>64$ & $>64$ & $>64$ & $>16$ & $>16$ & $>64$ & $>16$ & $<0.5$ & $<0.5$ & -ve \\
\hline C-19 & $>64$ & $>64$ & $>64$ & 8 & $>16$ & 8 & $>16$ & $<2$ & 2 & $+v e$ \\
\hline C-20 & $>64$ & $>64$ & $>64$ & $>16$ & $>16$ & 4 & $>16$ & $<0.5$ & $<0.5$ & $+v e$ \\
\hline C-21 & $>64$ & $>64$ & $>16$ & 8 & $>16$ & 16 & 4 & $<0.5$ & $<0.5$ & -ve \\
\hline C-22 & $>64$ & $>64$ & $>16$ & $>16$ & $>16$ & 16 & 4 & $<0.5$ & 2 & -ve \\
\hline C-23 & $>64$ & $>64$ & $>64$ & $>16$ & $>16$ & 64 & $>16$ & $<0.5$ & $<0.5$ & $+v e$ \\
\hline C-24 & $>64$ & $>64$ & $>16$ & 4 & $>16$ & 8 & 4 & $<2$ & $<0.5$ & $+v e$ \\
\hline C-25 & $>64$ & $>64$ & $>64$ & $>16$ & $>16$ & 16 & 4 & $<2$ & 2 & $+v e$ \\
\hline C-26 & $>64$ & $>64$ & $>64$ & 2 & 2 & 16 & 4 & $<0.5$ & $<0.5$ & $+\mathrm{ve}$ \\
\hline C-27 & $>64$ & $>64$ & $>64$ & $>16$ & $>16$ & 16 & 4 & $<0.5$ & $<0.5$ & -ve \\
\hline C-28 & $>64$ & $>64$ & $>16$ & $>16$ & $>16$ & 64 & $>16$ & $<2$ & 2 & -ve \\
\hline C-29 & $>64$ & $>64$ & $>16$ & $>16$ & $>16$ & 64 & $>16$ & $<0.5$ & $<0.5$ & -ve \\
\hline C-30 & $>64$ & $>64$ & $>64$ & 8 & $>16$ & 64 & $>16$ & $<0.5$ & $<0.5$ & $+v e$ \\
\hline \multirow[t]{2}{*}{ C-31 } & $>64$ & $>64$ & $>64$ & 8 & $>16$ & 16 & 4 & $<0.5$ & $<0.5$ & $+v e$ \\
\hline & $R=31$ & $R=31$ & $R=31$ & $R=31$ & $R=31$ & $\begin{array}{c}R=13 \\
(41.9 \%)\end{array}$ & $\begin{array}{c}R=16 \\
(51.6 \%)\end{array}$ & $\mathrm{R}=0$ & $\mathrm{R}=0$ & \\
\hline
\end{tabular}

CFZ=Ceftazidime, CTX=Ceftriaxone, CFP=Cefepime, IMP=Imipenem, MER=Meropenem, AK=Amikacin, GEN=Gentamicin, COL=Colistin, TIG=Tigecycline, Modified Hodge Test (MHT) 
Variable drug resistance pattern for cephalosporins and carbapenems was seen in participating hospitals (Table 1). In general, low rate of drug resistance was seen at Maternity and Children Hospital both for cephalosporins (8.1-10.8\%) and carbapenems (5.4\%). Among cephalosporins, high resistance to ceftriaxone (44.4-58\%) followed by (30-35.5\%) resistance to ceftazidime was seen at all participating hospitals. Among carbapenems, the high rate of resistance both to imipenem and meropenem was seen both at King Abdul Aziz hospital $(22.2 \%)$ and Al-Noor Specialist hospital (14.1\%), and low rates at other three hospitals (5.4-8.3\%).

\section{Minimum Inhibitory concentration of carbapenem resistant $K$. pneumoniae and results of Modified Hodge Test}

Out of $260 \mathrm{~K}$. pneumoniae isolates studied, 31 $(11.9 \%)$ showed $100 \%$ resistance to all cephalosporins and carbapenems tested (Table 1). Most of the strains exhibited high MIC values both for cephalosporins ( $\geq 64)$ and carbapenems $(\geq 8)$ indicating that these could be possible carbapenemase producers. Among aminoglycosides, resistance to amikacin was $41.9 \%$ and $51.6 \%$ to gentamicin. None of the isolates has shown resistance either to colistin or tigecycline (Table 2). Of these 31 isolates, fifteen isolates $(48.4 \%)$ were found positive for carbapenemase production (Figure 1 and Table 2) and 16 were negative using $\mathrm{MHT}$.

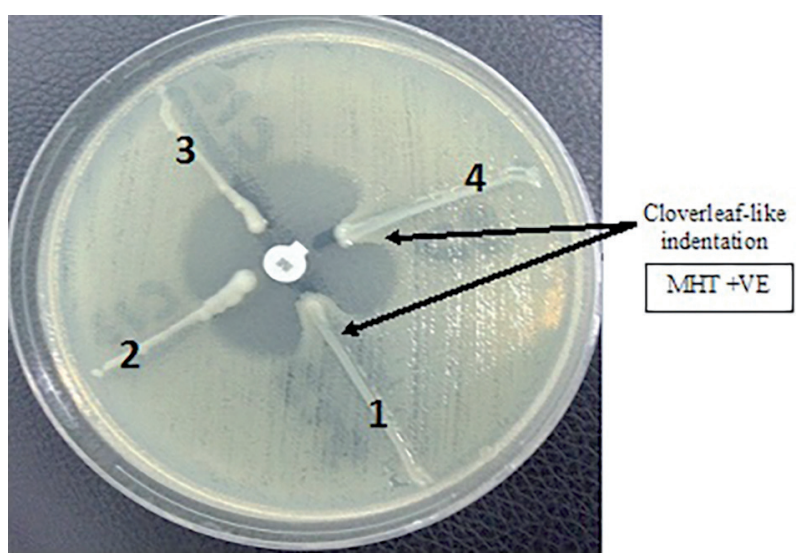

Figure 1. Modified Hodge Test for Carbapenemase Detection in Enterobacteriaceae.

\section{DISCUSSION}

In the last decade, the frequent occurrence of infections due to gram-negative organisms, particularly by $K$. pneumoniae resistant to third generation cephalosporins has resulted in the use of carbapen- ems for the treatment [20]. In the current study, 260 $K$. pneumoniae isolates showed drug resistance to third generation cephalosporin ranging from $29.2 \%$ to $42.7 \%$. These results are comparable to a study done in Latin America where resistance rate to third generation cephalosporins was found to be between $30-50 \%$ [21]. A study from Saudi Arabia also showed similar results of drug resistance: ceftriaxone $(31 \%)$, ceftazidime $(28 \%)$ and cefotaxime $(27 \%)$ and cefuroxime (27\%) [22].

Imipenem is frequently used to treat infections caused by multidrug-resistant $K$. pneumoniae; in particular those resistant to third generation cephalosporin. In our study, resistance to both imipenem and meropenem was $11.9 \%$. The resistance to meropenem showed in our study is comparable with the studies carried out in Turkey [23] where resistance to meropenem was $9 \%$. Regarding imipenem resistance, this study showed $11.9 \%$ resistance which is similar to reported earlier from Saudi Arabia (9\%) [22].

Production of carbapenemases is an increasing problem among clinical isolates of gram-negative bacilli. In particular, the spread of carbapenamse producing $K$. pneumoniae represents a serious threat to treatment protocols and has infection control implications [1]. Studies reported an increasing trend in the prevalence of carbapenemase producing K. pneumoniae from 2006 to 2010 [24] and continue to rise [7].

In Saudi Arabia, although carbapenem resistance remained uncommon among Enterobacteriaceae, outbreak due to carbapenem resistant organism has been reported. The first outbreak of carbapenems-resistant K. pneumoniae in Saudi Arabia was reported during 2010 in an intensive care unit of a tertiary care hospital, which involved 20 patients. The molecular basis of resistance was not reported [25]. However, in another study, investigators [15] detected OXA-48 and NDM enzymes in carbapenem resistant K. pneumoniae isolated at Riyadh hospitals in 2011. Recent study from Riyadh confirmed the emergence of OXA-48 and NDM-1 among $K$. pneumoniae in which 12 out of $60 \mathrm{~K}$. pneumoniae strains were NDM- 1 producers and $47 / 60$ strains were positive for OXA-48. No strain was positive for KPC [16]. Compared to above mentioned studies, current study reports a high percentage of $K$. pneumoniae $(48.4 \%)$ isolates to be carbapenemase producers determined by Modified Hodge Test (MHT). The limitation of this study is that the type of carbapenemase produced by these isolates cannot be determined and further characterization using 
molecular methods will detect the type of carbapenemase produced by these strains. However, it indicates that there is an upsurge of carbapenemase producing $K$. pneumoniae in these settings.

The antibiotic susceptibility profile of carbapenem resistant $K$. pneumoniae isolates of this study is similar to those reported for multidrug-resistant organisms producing carbapenemase from Saudi Arabia $[15,16]$ and other countries e.g., USA [26], Brazil [27] and China [28]. Patients infected with carbapenemase producing organisms are difficult to treat due to limited treatment options currently available e.g., tigecycline, colistin and fosfomycin, but these also have limitations [29]. Fortunately, our all carbapenemase producing strains were sensitive to both colistin and tigecycline. However, the resistance to colistin has recently been reported in K. pneumoniae [19]. These limitations warrant the need to search for new alternate treatment options.

The present study appears to be first from Makkah, Saudi Arabia to report carbapenemase producing $K$. pneumoniae from this area. Out of 31 potential carbapenemase producers, fifteen strains were found positive for carbapenemase production by MHT and of these; one strain (C-26) has exhibited reduced susceptibility to carbapenems $(2 \mu \mathrm{g} / \mathrm{ml})$ and was also found positive by MHT. This validates the findings that the isolate with reduced susceptibility to carbapenems can produce carbapenemase [30].

In conclusion, the present study reported that about $12 \%$ K. pneumoniae isolates were $100 \%$ resistant to both $3^{\text {rd }}$ generation cephalosporins and carbapenems and $48.4 \%$ were positive for carbapenemase production using MHT. Reporting of carbapenemase producing $K$. pneumoniae in this study necessitates the initiation of surveillance study both at local and national level to determine the actual prevalence of carbapenamse producing K. pneumoniae in Makkah and Saudi Arabia, respectively. Further characterization of these strains by molecular methods will determine the prevalent type of carbapenemase in this area.

\section{Acknowledgements}

The authors would like to thank Abeer Ibrahim AlZahrani, Ashwag Hashim Al-Zamur, Asma'a Mahmmod Asfour, Gofran Sharaf Al-Mosawi, Sultana Saad Al-Malki and Zainab Abdulhadi Al-Mubashir for their technical assistance. They all assisted in collection of samples and transportation from various hospitals and its processing in the laboratory.
Declaration of Conflicting Interests: The authors declare that they have no conflict of interest.

Financial Disclosure: No financial support was received.

\section{REFERENCES}

1. Nordmann P, Cuzon G, Naas T. The real threat of Klebsiella pneumoniae carbapenemase-producing bacteria. Lancet Infect Dis 2009; 9: 228-236.

2. World Health Organization. Antimicrobial resistance: global report on surveillance. Geneva: WHO Press 2014: 17-19.

3. Bush K, Jacoby GA. Updated Functional Classification of $\beta$-lactamases. Antimicrob Agents Chemother 2010; 54: 969976.

4. Nordmann P, Naas T, Poirel L. Global spread of carbapenemase-producing Enterobacteriaceae. Emerg Infect Dis 2011; 17:1791-1798.

5. Yigit H, Queenan AM, Anderson GJ, et al. Novel carbapenemhydrolyzing beta-lactamases, KPC-1, from a carbapenem resistant strains of Klebsiella pneumoniae. Antimicrob Agents Chemother 2001; 45:1151-1161.

6. Mammina C, Palma DM, Bonura C, et al. Outbreak of infection with Klebsiella pneumoniae sequence type 258 producing Klebsiella pneumoniae carbapenemase 3 in an intensive care unit in Italy. J Clin Microbiol 2010; 48:1506-1507.

7. Pesesky MW, Hussain T, Wallace M, et al. KPC and NDM-1 Genes in Related Enterobacteriaceae Strains and Plasmids from Pakistan and the United States. Emerg Infect Dis 2015; 21:1034-1037.

8. Tzouvelekis LS, Markogiannakis A, Psichogiou M, Tassios PT, Daikos GL. Carbapenemases in Klebsiella pneumoniae and other Enterobacteriaceae: an evolving crisis of global dimensions. Clin Microbiol Rev 2012; 25:682-707.

9. Yong D, Toleman MA, Giske CG, et al. Characterization of a new metallo-beta-lactamase gene, bla(NDM-1), and a novel erythromycin esterase gene carried on a unique genetic structure in Klebsiella pneumoniae sequence type 14 from India. Antimicrob Agents Chemother 2009; 253:5046-54.

10.Kumarasamy KK, Toleman MA, Walsh TR, et al. Emergence of a new antibiotic resistance mechanism in India, Pakistan, and the UK: a molecular, biological, and epidemiological study. Lancet Infect Dis 2010; 10:597-602.

11.Nordmann P, Poirel L, Walsh TR. The emerging NDM carbapenemases. Trends Microbiol 2011; 19:588-595.

12.Dortet L, Poirel L, Nordmann P. Worldwide dissemination of the NDM-type carbapenemases in gram-negative bacteria. Bio Med Res Int 2014; 1-12.

13. Dortet L, Poirel L, Al Yaqoubi F, Nordmann P. NDM-1, OXA48 and OXA-181 carbapenemase-producing Enterobacteriaceae in Sultanate of Oman. Clin Microbiol Infect 2012; 18 : E144-E148.

14. El-Herte RI, Araj GF, Matar GM, et al. Detection of carbapenem-resistant Escherichia coli and Klebsiella pneumoniae producing NDM-1 in Lebanon (case report). J Infect Dev Ctries 2012; 6:457-61.

15. Al-Agamy MH, Shibl AM, Elkhizzi NA, Meunier D, Turton JF Livermore DM. (2013) Persistence of Klebsiella pneumoniae clones with OXA-48 or NDM carbapenemases causing bacteraemias in a Riyadh hospital. Diagn Microbiol Infect Dis 76: 214-216. 
16. Shibl AM, Al-Agamy MH, Memish Z, Senok A, Khader SA, Assiri A. The emergence of OXA-48- and NDM-1-positive Klebsiella pneumoniae in Riyadh, Saudi Arabia. Int J Infect Dis 2013; 17:e1130-e1133.

17.Zowawi HM, Sartor AL, Balkhy HH, et al. Molecular characterization of carbapenemase producing Escherichia coli and Klebsiella pneumoniae in the countries of the Gulf Cooperation Council: dominance of OXA-48 and NDM producers. Antimicrob Agents Chemother 2014; 58:3085-3090.

18. Clinical and Laboratory Standard Institute. Performance standards for antimicrobial susceptibility testing; Twentysecond informational supplement. 2013; M100-S23: 33(1). Clinical Laboratory Standards Institute, Wayne, PA. USA

19. Capone A, Giannella M, Fortini D, et al. High rate of colistin resistance among patients with carbapenem-resistant Klebsiella pneumoniae infection accounts for an excess of mortality. Clin Microbiol Infect 2013; 19:E23-E30.

20. Paterson DL, Hujer KM, Hujer AM, et al. Extended spectrum beta-lactamases in $K$. pneumoniae blood stream isolates from seven countries: dominance and widespread prevalence of SHV- and CTX-M-type beta-lactamases. Antimicrob Agents Chemother 2003; 47:3554-60.

21. Sader HS and Sentry Participants Group (Sentry antimicrobial surveillance program report: Latin American and Brazilian results for 1997 through 2001. Braz J Infect Dis 2004; 8:25-79.

22. Ahmad S, Al-Juaid NF, Alenzi FQ, Mattar EH, Bakheet Oel-S. Prevalence, antibiotic susceptibility pattern and production of extended-spectrum beta-lactamases amongst clinical isolates of Klebsiella pneumoniae at Armed Forces Hospital in Saudi Arabia. J Coll Physicians Surg Pak 2009; 19:264-265.
23. Toroglu S, Keskin D. Antimicrobial resistance and sensitivity among isolates of Klebsiella pneumoniae from hospital patients in Turkey. Int J Agri Biol. 2011; 13: 941-46.

24. Zagorianou A, Silanou E, Losfidis E, et al. Microbiological and molecular characterization of carbapenemase producing Klebsiella pneumoniae endemic in a tertiary Greek hospital during 2004-2010. www.eurosurveillance.org 2012; 1-7.

25. Balkhy HH, El-Saed A, Al Johani SM, et al. The epidemiology of the first described carbapenem-resistant Klebsiella pneumoniae outbreak in a tertiary care hospital in Saudi Arabia: how far do we go? Eur J Clin Microbiol Infect Dis. 2012; 31:1901-1909.

26. Rasheed JK, Kitchel B, Zhu W, et al. New Delhi metallo- $\beta$ lactamase-producing Enterobacteriaceae, United States. Emerg Infect Dis 2013; 19:870-78.

27. Rozales FP, Ribeiro VB, Magagnin CM, et al. Emergence of NDM-1-producing Enterobacteriaceae in Porto Alegre, Brazil. Int J Infect Dis 2014; 25:79-81.

28. Wang X, Chen G, Wu X, et al. Increased prevalence of carbapenem resistant Enterobacteriaceae in hospital setting due to cross-species transmission of the blaNDM-1 element and clonal spread of progenitor resistant strains. Front Microbiol 2015; 6:595.

29. Kanj SS and Kanafani ZA. Current concepts in antimicrobial therapy against resistant gram-negative organisms: Extended-Spectrum $\beta$-lactamase-producing Enterobacteriaceae carbapenem-resistant Enterobacteriaceae, and multidrug resistant Pseudomonas aeruginosa. Mayo Clinic Proceedings 2011; 86:250-59.

30. Endimiani A, Perez F, Bajaksouzian S, et al. Evaluation of updated interpretative criteria for categorizing Klebsiella pneumoniae with reduced susceptibility. J Clin Microbiol 2010; 48:4417-4425. 